Portland State University

PDXScholar

Electrical and Computer Engineering Faculty

Publications and Presentations

Electrical and Computer Engineering

$1-1-2011$

\title{
Extraction of Small Boat Harmonic Signatures from Passive Sonar
}

\author{
George Lloyd Ogden \\ Portland State University \\ Lisa M. Zurk \\ Portland State University, zurkl@pdx.edu \\ M. E. Jones \\ Pacific Northwest National Laboratory \\ M. E. Peterson \\ Pacific Northwest National Laboratory
}

Follow this and additional works at: https://pdxscholar.library.pdx.edu/ece_fac

Part of the Electrical and Computer Engineering Commons

Let us know how access to this document benefits you.

\section{Citation Details}

Ogden, G. L., Zurk, L. M., Jones, M. E., \& Peterson, M. E. (2011). Extraction of small boat harmonic signatures from passive sonar. The Journal of the Acoustical Society of America, 129, 3768.

This Article is brought to you for free and open access. It has been accepted for inclusion in Electrical and Computer Engineering Faculty Publications and Presentations by an authorized administrator of PDXScholar. Please contact us if we can make this document more accessible: pdxscholar@pdx.edu. 


\title{
Extraction of small boat harmonic signatures from passive sonar
}

\author{
George L. Ogden ${ }^{\text {a) }}$ and Lisa M. Zurk \\ Northwest Electromagnetics and Acoustics Research Laboratory, Portland State University, P.O. Box 751, \\ Portland, Oregon 97207 \\ Mark E. Jones and Mary E. Peterson \\ Pacific Northwest National Laboratory, P.O. Box 999, Richland, Washington 99352
}

(Received 8 October 2010; revised 26 March 2011; accepted 28 March 2011)

\begin{abstract}
This paper investigates the extraction of acoustic signatures from small boats using a passive sonar system. Noise radiated from a small boats consists of broadband noise and harmonically related tones that correspond to engine and propeller specifications. A signal processing method to automatically extract the harmonic structure of noise radiated from small boats is developed. The Harmonic Extraction and Analysis Tool (HEAT) estimates the instantaneous fundamental frequency of the harmonic tones, refines the fundamental frequency estimate using a Kalman filter, and automatically extracts the amplitudes of the harmonic tonals to generate a harmonic signature for the boat. Results are presented that show the HEAT algorithms ability to extract these signatures.
\end{abstract}

(C) 2011 Acoustical Society of America. [DOI: 10.1121/1.3583500]

PACS number(s): 43.60.Hj, 43.60.Lq, 43.30.Wi [EJS]

Pages: $3768-3776$

\section{INTRODUCTION}

The automated detection and classification of maritime traffic is a challenging problem and is of great importance to many organizations. For marine protected areas (MPAs), an automated boat detection system could alert authorities of vessel traffic. However, in some MPAs commercial snorkeling and diving boats are authorized while fishing vessels are not. For this reason, a classification system is needed to discriminate these different types of boats. The need for similar systems arises in the monitoring of harbor traffic for national security. There are many different methods for boat detection, examples including radar, ${ }^{1}$ electro-optic (EO) and infrared (IR) cameras, ${ }^{2}$ and both active and passive sonar. Active sonar and radar provide little additional information beyond detection. Radar and optical methods are limited by line of sight for detection, and optical systems can be obscured by rain, fog, or may require daylight. Active sonar can be used for detection of quiet targets, but the high level of reverberation in shallow water environments often causes many false detections, which limits its utility.

As an alternative, passive sonar has been proven to be an efficient tool for the detection and identification of selfemitting targets. ${ }^{3,4}$ Passive sonar has been used to detect SCUBA divers by observing peaks in the frequency energy distribution due to the divers breaths. ${ }^{5}$ The breathing rate and the spectrum intensity give information of the range of the diver. The same research group used passive sonar to observe the radiated spectrum of small boats and other harbor traffic and investigated the effects of boat noise on the detection range of divers. ${ }^{6}$ While passive sonar was used here to examine the sound emitted from boats, this work is mainly focused on the detection of divers using passive sonar. There has yet to be any significant work on classification of small boats in the literature.

${ }^{\text {a)} E l e c t r o n i c ~ m a i l: ~ o g d e n g l @ g m a i l . c o m ~}$
This paper is focused on the extraction of acoustic signatures from small boats using a passive sonar system. Passive spectra of boats include broadband noise as well as tonals due to the harmonics of the engine speed and shaft/ propeller rotation. $^{7}$ The algorithm developed here extracts the harmonic features to facilitate the exploration of the relationship between these features and the identification of specific boats. These features consist of harmonic amplitudes, SNRs, and the fundamental frequencies of the boat noise.

Fundamental frequency estimation is a topic that spans many disciplines including speech recognition, ${ }^{8}$ biomedical signal processing, ${ }^{10}$ and musical pitch estimation. ${ }^{11}$ In Refs. 12-14, least squares estimators were used to determine fundamental frequency of harmonic sinusoidal signals. In Ref. 8 , speech pitch was estimated by maximizing the cross correlation of a speech signal over a range of feasible pitch values. An adaptive comb filtering technique was used in Ref. 9 to estimate fundamental frequency of noisy harmonic signals. A marginalized particle filter was used in Ref. 11 to estimate and to track the fundamental frequency, and in this case, multiple fundamental frequencies, of musical signals. In Ref. 10, a marginalized particle filter was also used to estimate and to track the instantaneous frequency of two biomedical signals: electrocardiogram and arterial blood pressure.

In this paper a Harmonic Extraction and Analysis Tool (HEAT) has been designed to estimate the fundamental frequency of the harmonic content generated by the engine and propeller of small boats. A discrete Kalman filter is applied to refine the estimated fundamental frequency and create a track through time. The Kalman filter is a widely used recursive algorithm used to estimate the mean and error covariance of a state through time given a series of noisy observations of the state. ${ }^{15}$ Kalman filter and its extended forms have been applied to many areas in acoustic signal processing. In Ref. 16, an extended Kalman filter is used to track objects in a multistatic active sonar geometry. 
In Ref. 17, a Kalman filter was applied in real time speech processing to find a model that fits the natural speech.

Harmonics of the fundamental frequency are extracted, and the amplitudes of each harmonic are used as signatures of the boat. Extracted signatures from data collected in Sequim Bay, WA are presented. These examples quantify the algorithms ability to automatically extract these harmonic signatures for later use in classification.

\section{SIGNAL MODEL AND DIGITAL PROCESSING}

Many researchers have studied radiated noise from large ships, both by modeling and measurement. In the 1970s, Gray and Greely ${ }^{18}$ developed a model to predict source level and frequency of the acoustic energy generated by propeller cavitations. In the 1990 s, Arveson and Vendettis ${ }^{19}$ conducted a series of measurements of the noise radiated from the M/V Overseas Harriette and found agreement with Gray and Greeley's model. These references characterize the radiated noise from large ships quite well. However, much less work has been done to characterize the radiated noise from small vessels.

Ross $^{3}$ and Urick ${ }^{4}$ have given an excellent description of radiated noise from large surface ships and submarines. They have shown that the radiated noise from a ship is a combination of broadband noise and sinusoidal tonal signals. The broadband noise is generated by many sources including propeller cavitations and impulsive events in the engine. This broadband noise propagates through the water, and when received on a hydrophone, generates the classical bathtub pattern that is often associated with passive acoustic signatures. This bathtub pattern is due to the different multipath arrivals of the noise adding up in and out of phase. The sinusoidal tonal signals can be related to details about the ships engine and propeller. They are also the fundamental components of a harmonic set. Table I shows the major contributions to the tonals from the ships engine and propeller. The model of radiated ship noise represented as a sum of broadband noise and tonal frequencies is used to describe the noise radiated from small boats.

\section{A. Signal model}

Consider a sum of many periodic sinusoidal signals whose frequencies are all harmonically related, being integer

TABLE I. Fundamental frequencies from the engine and propeller.

\begin{tabular}{lr}
\hline \hline \multicolumn{1}{c}{ Engine Rates } & Propeller Rates \\
\hline Cylinder Firing Rate & Shaft Rotation Rate \\
$f_{C F}=f_{C R} / 2$ & $f_{S R}=f_{C R} / \Lambda_{g}$ \\
& $\Lambda_{g}=$ Gear Ratio \\
Crankshaft Rotation Rate & Blade Rotation Rate \\
$f_{C R}=R P M / 60$ & $f_{B R}=N_{b} f_{S R}$ \\
RPM $=$ Engine Speed & $N_{b}=$ Number of Blades \\
Engine Firing Rate & \\
$f_{E F}=N_{C} f_{C F}$ & \\
$N_{c}=$ Number of Cylinders & \\
\hline \hline
\end{tabular}

multiples of a fundamental frequency. This signal can be written as follows:

$$
s(t)=\sum_{h=1}^{H} A_{h} \cos \left(2 \pi h \gamma t+\varphi_{h}\right),
$$

where $h$ is the harmonic number, $A_{h}$ and $\varphi_{h}$ are the amplitude and phase of the $h$ th harmonic component, and $\gamma$ is the fundamental frequency. Assume that this signal is of infinite length. The Fourier transform of $s(t)$ consists of a series of delta functions with even spacing $\gamma$.

Now consider the noise radiated from a ship as a combination of broadband noise and harmonically related sinusoidal tonal signals. This can be written as:

$$
\begin{aligned}
r(t) & =s(t)+n(t) \\
& =\sum_{h=1}^{H} A_{h} \cos \left(2 \pi h \gamma t+\varphi_{h}\right)+n(t) .
\end{aligned}
$$

In this equation the fundamental frequency and harmonic amplitudes are unknown. This fundamental frequency $\gamma$ is related to the engine speed and other parameters by Table I. We can rewrite $r(t)$ as $r(t, \theta)$, where the value $\theta$ represents a set of estimation parameters including the fundamental frequency, $\gamma$, as well as the amplitude of all the harmonics, $A_{h}$, thus $\theta=\left\{\gamma, A_{h}\right\}$.

The signal received on the hydrophone is different from the signal radiated from the boat for a number of reasons including changes in engine speed, Doppler shift, and other propagation effects. The received signal can still be modeled as a sum of sinusoidal signals as in (1), but with a timevarying fundamental frequency $\gamma$. Hence, $\gamma$ is written as $\gamma(t)=f_{o}+\Delta f(t)$ where $f_{o}$ is the fundamental frequency, and $\Delta f(t)$ is the change in fundamental frequency over time. The quantity $r(t, \theta)$ can finally be written as:

$$
\begin{aligned}
r(t, \theta) & =s(t, \theta)+n(t) \\
& =\sum_{h=1}^{H} A_{h} \cos \left(2 \pi h \gamma(t) t+\varphi_{h}\right)+n(t) .
\end{aligned}
$$

\section{B. Digital processing}

The signal received on the hydrophone, $r(t, \theta)$, is a continuous signal with time-varying frequency content. The signal is first digitally sampled before being processed. A timefrequency transform is then applied to the signal to show the time-varying frequency content. The Short-time Fourier Transform (STFT) is computed by moving a short window along the data (creating a "snapshot") and computing the Fourier transform of the data along that window. The window length is assumed to be short enough that the change in fundamental frequency within the window is negligible, i.e., $\Delta f(t) \sim \Delta f\left(t_{k}\right)$ within the window. Now $\gamma_{k}=f_{o}+\Delta f\left(t_{k}\right)$, where $t_{k}$ is the center time for the $k$ th snapshot.

The signal in frequency domain for each window is the convolution of the Fourier transformed data with the Fourier 
transform of the time-domain window, $S(f, \theta)=\mathscr{F}\{s(t$, $\theta)\} * \mathscr{F}\{$ rectwin $(t)\}$, which results in $S(f, \theta)$ being a summation of weighted $\operatorname{sinc}(\operatorname{sinc} x \equiv \sin x / x)$ functions:

$$
S\left(f, \theta_{k}\right)=\sum_{h=1}^{H} \frac{A_{h}}{2} \operatorname{sinc}\left[\pi\left(f-h \gamma_{k}\right)\right] .
$$

Suppose $r(t, \theta)$ to be of finite length $T_{r}$. The signal is sampled at frequency $f_{s}$, with a sampling period $\Delta t=1 / f_{s}$. Thus $r(t, \theta)$ can be written as $r(j \Delta t, \theta)$ where $j=\{0$, $\left.1, \cdots, N_{r}-1\right\}$ and $N_{r}=T_{r} / \Delta t$. Then the signal is partitioned into $K$ overlapping segments, or snapshots, where each snapshot is of length $T$ seconds, or $N_{s}$ samples where $N_{s}=T / \Delta t$. The notation $r_{k}\left(n \Delta t, \theta_{k}\right)$ is used to represent the $n$th sample of the $k$ th snapshot. The received data, $r(n \Delta t, \theta)$, is now of dimension $\left[N_{s} \times K\right]$. Each snapshot is then transformed to the frequency domain by computing the $N_{s}$-point DFT using an FFT algorithm:

$R_{k o}\left(m \Delta f, \theta_{k}\right)=\frac{2}{N_{s}} \sum_{n=-N_{s} / 2}^{N_{s} / 2-1} r_{k}\left(t_{k}+n \Delta t, \theta_{k}\right) e^{-j m 2 \pi \Delta f n \Delta t}$

where $t_{k}$ is the center time for the $k$ th snapshot and $R_{k o}\left(m \Delta f, \theta_{k}\right)$ is the DFT coefficient at the frequency bin $m \Delta f$. The frequency resolution, or width of the bin $\Delta f$, is determined by the length of the snapshot window such that $\Delta f=1 / T$. For simplicity let $f_{m} \equiv m \Delta f$, where $f_{m}$ $=\Delta f\left\{-N_{s} / 2, \cdots,-1,0,1, \cdots, N_{s} / 2-1\right\}, \quad$ so $\quad R_{k o}(m \Delta f$, $\left.\theta_{k}\right)=R_{k o}\left(f_{m}, \theta_{k}\right)$, which is of dimension $\left[N_{s} \times K\right]$.

Lastly, once the STFT is computed, each snapshot is normalized along frequency. The signal that is received is a combination of the tonals from the engine and propeller, broadband noise, and any environmental noise in the area. Since this noise is generally non-Gaussian, the received signal is normalized using a moving window of length $W$ along frequency.

$$
R_{k}\left(f_{m}, \theta_{k}\right)=\frac{R_{k o}\left(f_{m}, \theta_{k}\right)-\mu_{R, W}}{\sigma_{R, W}},
$$

where $\mu_{R, W}$ and $\sigma_{R, W}$ are the mean and standard deviation of $R_{k o}\left(f_{m}, \theta_{k}\right)$ in the window $\mathrm{W}$.

\section{HARMONIC EXTRACTION AND ANALYSIS TOOL (HEAT)}

The overall goal is to develop a boat detection and signature extraction algorithm that can be implemented in realtime on passive acoustic systems. Figure 1 shows the structure of the developed approach. The first block shows data collection and pre-processing, as discussed in the previous section. The next block is an event detection algorithm, which is used to pick out segments of acoustic data where a ship signature is present. Once a boat signature has been detected, certain parameters about the boat need to be estimated in order to give some kind of information that will help in identifying the type of boat. The Harmonic Extraction and Analysis Tool (HEAT) has been designed to extract

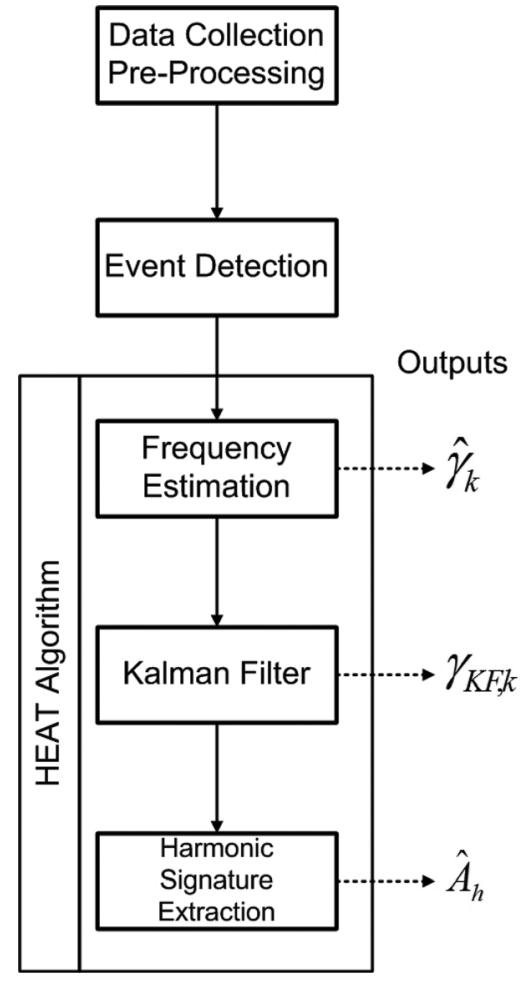

FIG. 1. General overview of detection and estimation algorithm.

important information from the data for later use in classification.

There are three main steps to the HEAT algorithm. The first step is to estimate the fundamental frequency of the harmonic content from the boat. The method used in this paper is a frequency domain method similar to the maximum likelihood method described in Ref. 20. In their research, a time domain method for estimating the pitch period of voiced speech based on a maximum likelihood formulation. The frequency domain analog to that method matches a comb-like filter to the autocorrelation of the periodic signal. The Fourier transform of a signal is correlated to a comb filter to generate an estimate of the fundamental frequency. This puts the frequency in a linear space, so a Kalman filter can then be used to track the fundamental frequency through time. The time evolving fundamental frequency estimated from the Kalman filter is used as a basis for extracting the amplitude of the harmonic tonals from the data. These harmonic amplitudes are what make up the harmonic signature.

\section{A. Fundamental frequency estimation}

The noise radiated from small boats is modeled as a periodic, multi-harmonic signal, as described in Sec. 2.1. In this section, a method of estimating the fundamental frequency of a harmonic set is described. The Pearson Product-Moment Correlation Coefficient (PMCC) is used to estimate the correlation of the unknown harmonic set to a signal with known fundamental frequency.

The PMCC is a measure of linear association between two random variables. ${ }^{21}$ Consider an ordered pair of random variables $X$ and $Y$ with mean $\mu_{x}$ and $\mu_{y}$, standard deviation $\sigma_{x}$ 
and $\sigma_{y}$, and covariance $\sigma_{x y}$. The correlation coefficient between $X$ and $Y$ is defined as

$$
\rho=\frac{\sigma_{x y}}{\sigma_{x} \sigma_{y}}
$$

where $\rho$ is bounded between -1 and 1 . This can be estimated from a sample of $X$ and $Y$ by

$$
\hat{\rho}=\frac{\sum_{i}\left(x_{i}-\mu_{x}\right)\left(y_{i}-\mu_{y}\right)}{\sqrt{\sum_{i}\left(x_{i}-\mu_{x}\right)^{2}} \sqrt{\sum_{i}\left(y_{i}-\mu_{y}\right)^{2}}},
$$

The variable $\hat{\rho}$ will be used to indicate an estimate of the correlation coefficient. The PMCC is used in this algorithm to measure the similarity of the measured signal $R_{k}\left(f_{m}, \theta_{k}\right)$ to a signal with known parameters.

\section{Signal replica model}

In (3) the tonals recorded on the hydrophone were modeled as a sum of weighted, harmonically related sinc functions, with fundamental frequency $\gamma_{k}$ at time $t_{k}$. This same model is used to generate comb filters which are correlated to the received signal, only here it is assumed that all the harmonics have equal amplitude:

$$
\begin{aligned}
C_{m p} & =S\left(f_{m}, \theta_{k} \mid \gamma_{k}=\zeta_{p}, A_{h}=2\right) \\
& =\sum_{h=1}^{H} \operatorname{sinc}\left[\pi\left(f_{m}-h \zeta_{p}\right)\right],
\end{aligned}
$$

where $C_{m p}$ is a row in the matrix $\overline{\bar{C}}$. The matrix $\overline{\bar{C}}$ is dimension $\left[N_{\gamma} \times N_{s}\right]$, where the fundamental frequency is discretized into $N_{\gamma}$ points of width $\Delta \gamma$. The variable $\zeta$ gives the fundamental frequency of each comb filter. It is indexed by $p$ such that $\zeta_{p}=\gamma_{\min }+p \Delta \gamma$.

\section{Fundamental frequency estimation using PMCC}

To estimate the fundamental frequency of the signal received at time $t_{k}, R_{k}\left(f_{m}, \theta_{k}\right)$, the signal is compared with a signal replica model (7) using the PMCC (6):

$$
\hat{\rho}_{p k}\left(\zeta_{p}, t_{k}\right)=\frac{\sum_{f m}\left(C_{m p}-\mu_{C}\right) R_{k}\left(f_{m}, \theta_{k}\right)}{\sqrt{\sum_{f m}\left(C_{m p}-\mu_{C}\right)^{2}} \sqrt{\sum_{f m} R_{k}\left(f_{m}, \theta_{k}\right)^{2}}} .
$$

Note that the mean of $R_{k}\left(f_{m}, \theta_{k}\right)$ does not appear in (8) since it is already zero mean by Eq. (5). The value $\hat{\rho}_{p k}\left(\zeta_{p}, t_{k}\right)$ is the correlation coefficient of the $k$ th snapshot of $R_{k}\left(f_{m}, \theta_{k}\right)$ to a comb filter with fundamental frequency $\zeta_{p}$. This is done for each snapshot which makes the matrix $\overline{\bar{\rho}}$ dimension $\left[N_{\gamma} \times K\right]$. For brevity, we will drop the variables from $\hat{\rho}_{p k}\left(\zeta_{p}, t_{k}\right)$, i.e., $\hat{\rho}_{p k} \equiv \hat{\rho}_{p k}\left(\zeta_{p}, t_{k}\right)$.

When the fundamental frequency in $C_{m p}$ is equal to the fundamental frequency in $R_{k}\left(f_{m}, \theta_{k}\right)$, i.e., $\zeta_{p}=\gamma_{k}, \hat{\rho}_{p k}$ results in a high correlation and a peak in $\overline{\bar{\rho}}$. This peak frequency is denoted as $\hat{\gamma}_{k}$. Also, if the spacing in the replica signal is twice the fundamental frequency of the received signal, it generates another peak in $\overline{\bar{\rho}}$ at that frequency. In fact, there

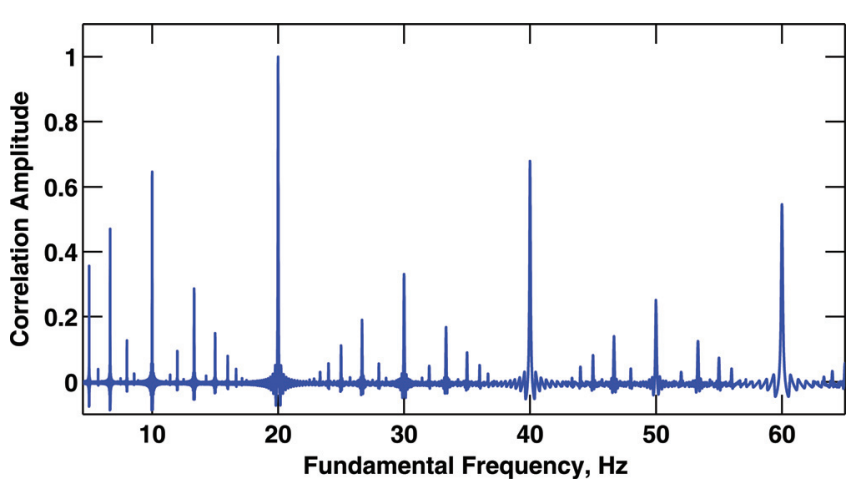

FIG. 2. (Color online) The fundamental frequency correlation in (8) for the replica signal with a fundamental frequency of $20 \mathrm{~Hz}$ shows a peak indicating perfect correlation at $20 \mathrm{~Hz}$, as well as several partial matches.

are many peaks that show up in $\overline{\bar{\rho}}$ as a result of partial matches with multiples of the fundamental frequency. This is demonstrated in Figure 2, which shows the result the PMCC analysis of $\overline{\bar{C}}$ to $S\left(f_{m}, \theta_{k} \mid \gamma_{k}=20, A_{h}=2\right)$.

The peak at $20 \mathrm{~Hz}$ shows the perfect correlation of the replica signal with fundamental frequency of $20 \mathrm{~Hz}$ to itself. There are many other peaks that give high correlation due to only a fraction of the peaks lining up with the model. It is for this reason, as well as the desire to track the changing frequency content through time, that a Kalman filter is applied to $\overline{\bar{\rho}}$. Each of the peaks in $\overline{\bar{\rho}}$ and their associated frequencies $\hat{\gamma}_{k}$ will be combined into a set of detections $Z_{k}$ to be used as measurements for the Kalman filter.

\section{B. Kalman filter}

The fundamental frequency estimator in (8) results in a correlation of the measured signal $R_{k}\left(f_{m}, \theta_{k}\right)$ with a replica signal $C_{m p}$ for all possible fundamental frequencies $\zeta_{p}$ and for every snapshot $k$. A simple way to estimate the fundamental frequency at time $k$ is to find the value of $\zeta_{p}$ where $\hat{\rho}_{p k}$ is maximum $\hat{\gamma}_{k}=\arg \max _{p} \hat{\rho}_{p k}$. However, it is not enough since multiple signals of interest (boats) can be present with different fundamental frequencies. Also, if the gear ratio in the engine is not an integer number, the fundamental frequency for the engine harmonics will be different from the fundamental frequency of the shaft and propeller harmonics. For that reason, a Kalman filter has been implemented in the correlation domain that will allow for a time evolving estimate of the fundamental frequency for each of the peaks present.

The Kalman filter works by projecting the mean $\hat{\Gamma}_{k}^{-}$and error covariance $P_{k}^{-}$of the state forward in time from time $t_{k-1}$ to time $t_{k}$ from initial estimates supplied to the filter, $\hat{\Gamma}_{k-1}$ and $P_{k-1} \cdot{ }^{15}$

$$
\begin{gathered}
\text { Predict } \\
\hat{\Gamma}_{k}^{-}=F \hat{\Gamma}_{k-1} \\
P_{k}^{-}=F P_{k-1} F^{T}+Q,
\end{gathered}
$$

where $F$ is the state transition matrix and $Q$ is the process noise covariance matrix. The estimate of the mean $\hat{\Gamma}_{k}$ and error covariance $P_{k}$ are then corrected, or updated (10), based on the measurement of the state $z_{k}$ at time $t_{k}$. 
Update

$$
\begin{aligned}
K_{k} & =P_{k}^{-} H^{T}\left(H P_{k}^{-} H^{T}+R\right)^{-1} \\
\hat{\Gamma}_{k} & =\hat{\Gamma}_{k}^{-}+K_{k}\left(z_{k}-H \hat{\Gamma}_{k}^{-}\right) \\
P_{k} & =\left(I-K_{k} H\right) P_{k}^{-}
\end{aligned}
$$

where $K_{k}$ is the Kalman gain at time $t_{k}, R$ is the measurement noise covariance matrix, and $H$ relates the state to the measurement. At the next time step the corrected estimates are fed back into the prediction equations and the process repeats. This gives a filtered estimate of the mean and error covariance of the state for all times which forms a track.

\section{Fundamental frequency tracking using Kalman filter}

A discrete Kalman filter has been implemented as a peak follower that tracks the PMCC in (8). The tracker adapts a near constant velocity process model to tracking frequency, given by

$$
\begin{aligned}
\Gamma_{k} & =F \Gamma_{k-1}+w_{k}, \\
\Gamma_{k} & =\left[\begin{array}{c}
\gamma_{K F, k} \\
\dot{\gamma}_{K F, k} \\
\rho_{K F, p k}
\end{array}\right], \\
F & =\left[\begin{array}{lll}
1 & 1 & 0 \\
0 & 1 & 0 \\
0 & 0 & 1
\end{array}\right], \\
w_{k} & \sim N(0, Q),
\end{aligned}
$$

where $\Gamma_{k}$ is the state vector for a single track, $\gamma_{K F, k}$ and $\dot{\gamma}_{K F, k}$ represent the frequency and change in frequency (frequency velocity) from time $t_{k-1}$ to $t_{k}$, respectively, $\rho_{K F, p k}$ is the correlation value for frequency $\gamma_{K F, k}, F$ is the state transition matrix, and $w_{k}$ is assumed to be a zero-mean white Gaussian process with variance Q.

For a Kalman filter tracking an object's position, the near constant velocity model would assume that from time $t_{k-1}$ to $t_{k}$, the change in velocity of the object is negligible. The position can be predicted as the previous position plus some change in position due to the object moving at some velocity over some time step. The adaptation of this model to tracking frequency assumes that the change in frequency from time $t_{k-1}$ to $t_{k}$ is negligible, so the frequency at time $t_{k}$ can be predicted as $\gamma_{K F, k-1}+\dot{\gamma}_{K F, k}$. Actual changes to the frequency are accounted for through the process noise term.

The measurement model directly relates the measurement $z_{k}$ to the state at time $t_{k}$ as follows:

$$
\begin{aligned}
& z_{k}=H \Gamma_{k}+v_{k}, \\
& z_{k}=\left[\begin{array}{c}
\hat{\gamma}_{k} \\
\hat{\rho}_{p k}
\end{array}\right], \\
& H=\left[\begin{array}{lll}
1 & 0 & 0 \\
0 & 0 & 1
\end{array}\right], \\
& v_{k} \sim N(0, R),
\end{aligned}
$$

where $z_{k}$ is the measurement with frequency $\hat{\gamma}_{k}$ and correlation value $\hat{\rho}_{p k}, H$ relates the state to the measurement, and $v_{k}$ is assumed to be a zero-mean white Gaussian process with variance $\mathrm{R}$.

The process noise covariance $\mathrm{Q}$ and the measurement noise covariance $\mathrm{R}$ are both matrices defined as follows:

$$
\begin{aligned}
& Q=\left[\begin{array}{ccc}
Q_{\gamma} & 0 & 0 \\
0 & Q_{\dot{\gamma}} & 0 \\
0 & 0 & Q_{\rho}
\end{array}\right], \\
& R=\left[\begin{array}{cc}
R_{\gamma} & 0 \\
0 & R_{\rho}
\end{array}\right] .
\end{aligned}
$$

These parameters are the main tuning parameters of the Kalman filter. The amount of noise injected into the process or measurement model dictates the certainty with which the models are trusted to estimate the state.

\section{Tracker logic}

The Kalman filter requires an initial estimate of the state, so the tracking algorithm includes logic-based track initiation and termination. ${ }^{16}$ There are several possible fundamental frequencies that fit the data, which are obtained by applying a threshold, $\lambda_{\gamma}$, to the fundamental frequency estimate, $\overline{\bar{\rho}}$, for each snapshot. These frequencies are presented to the tracker as a set of detections or observations for time $t_{k}$ in the set $Z_{k}$. These detected frequencies are used to initiate tracks, as well as measurements for the Kalman filter. There are three states which a track can be in:

- initiated-if a fundamental frequency is detected in $M$ out of $N$ consecutive snapshots, a track is created,

- flagged-if a track has no associated observations in the set $Z_{k}$, the track is flagged for termination,

- terminated-if a track is flagged for $N_{F}$ consecutive snapshots, the track is terminated.

At each time step, a set of observations need to be paired to the tracks. For an observation to be associated with a track the observation must satisfy the following threshold condition:

$$
\left(z_{k}-H \hat{\Gamma}_{k}^{-}\right)\left(H P_{k}^{-} H^{T}+R\right)^{-1}\left(z_{k}-H \hat{\Gamma}_{k}^{-}\right)^{T}<\chi^{2}
$$

The $\chi^{2}$ value gives a measurement of the difference between each observed frequency and the frequency predicted by the Kalman filter. A small value of $\chi^{2}$ represents a closer match between the observed and predicted frequencies. In the case where multiple observations satisfy this condition, the best match is the one with the smallest $\chi^{2}$ value.

Before a track is initiated, the algorithm searches within a small window, $W_{\gamma}$, around the proposed frequency for any existing tracks. If a track already exists within that window, the new track is immediately terminated, as it is assumed to belong to the already existing track. The $W_{\gamma}$ window keeps the number of duplicate tracks within a preferred limit. The more tracks the Kalman filter computes, the slower the algorithm would run. The logic initiates more tracks with smaller 
window size. On the other hand, the larger the window, the more likely that a real track nearby an existing track would not be initiated.

\section{Harmonic content parameter}

The Kalman filter outputs multiple tracks, each track being a time-evolving estimate of a fundamental frequency for the harmonic content in the signal $R_{k}\left(f_{m}, \theta_{k}\right)$. To determine which track best fits the data, a parameter $\Psi$ is calculated from the estimate of the correlation, which is the third component of the state vector in (11), as follows:

$$
\Psi[\kappa]=\sqrt{\frac{1}{L} \sum_{k=1}^{L}\left|\rho_{K F, p k}[\kappa]\right|^{2}},
$$

where $\kappa$ is the index of the track, $\rho_{K F, p k}[\kappa]$ is the estimate of the amplitude from the Kalman filter for track $\kappa$, and $\mathrm{L}$ is the length of the track in snapshots. The track with the highest $\Psi$ value is chosen as the best fit to the data and is considered to be the best estimate of the fundamental frequency.

The value $\Psi$ is a measure of how well the data (5) fits the signal replica model (7). A value of $\Psi$ equal to one would mean that the data perfectly matches the model. Since the signal replica model assumes that all the harmonics are present and equal in amplitude, and that there is no noise present, this perfect match of measurement to model is not achievable. The value $\Psi$ can also be described as a measure of how much harmonic content is present in the signal. Since the parameter $\Psi[\kappa]$ is the average of $\rho_{K F, p k}$ over the length of the track, the higher $\Psi[\kappa]$ is, the more harmonics will be visible in the spectrogram over the length of the track. The opposite case is also true in that the lower $\Psi[\kappa]$ is, the less harmonic content will be visible in the spectrogram.

\section{Harmonic signature extraction}

The harmonic signature is considered to be the "acoustic fingerprint" of a boat. Table I describes the fundamental frequencies generated by a motor expressed in terms of engine speed, number of cylinders, gear ratio, number of blades, etc., which are fundamental frequencies of the tonals described in (2). In the previous sections the fundamental frequency $\gamma_{k}$ was estimated and tracked through time using a Kalman filter, $\gamma_{K F, k}$. This estimate of $\gamma_{k}$ is the first part of the estimation parameter $\theta$. The second part is the amplitude of all the harmonics, $A_{h}$. These harmonic amplitudes are called the harmonic signature of a boat.

The fundamental frequency track $\gamma_{K F, k}$ that best fits the data is determined by the $\Psi$ parameter. To obtain a harmonic signature, the best fitting track is first projected onto the spectrogram at all harmonic frequencies. Figure 3 illustrates the projection of the fundamental frequency track. Figure 3(a) shows the spectrogram of a short segment (100 seconds) of recorded boat noise. Figure 3(b) shows the result of projecting the fundamental frequency track onto the spectrogram for all the harmonics. The $\mathrm{x}$-axis was truncated to show only $800-1400 \mathrm{~Hz}$ to better illustrate the projection.
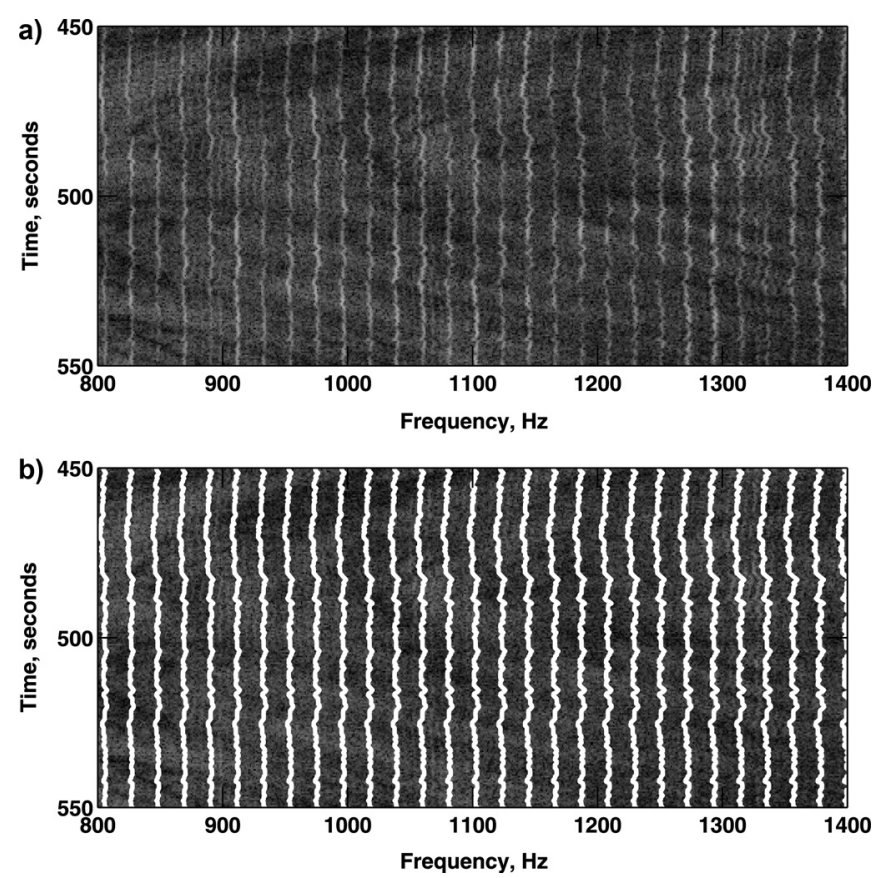

FIG. 3. (a) Spectrogram showing a $100 \mathrm{~s}$ segment of a recorded boat signature recorded in Sequim Bay, WA in the frequency range of $800 \mathrm{~Hz}$ to $1400 \mathrm{~Hz}$. (b) The projections of the fundamental frequency track overlaid on the spectrogram (white)

Projecting the track onto the spectrogram is achieved by finding the frequency bins $f_{m}$ closest to integer multiples of $\gamma_{K F, k}$ for each snapshot. The amplitude for each of the harmonics is then found by searching for a peak in $R_{k o}\left(f_{m}, \theta_{k}\right)$ within a small window around the projected frequencies, resulting in the estimate of the amplitude for each harmonic $\hat{A_{h}}$. The local noise of each harmonic is also estimated by averaging the spectrogram in a small window on each side of the peak. The window size is sensitive to the specific nature of the data, specifically to the dynamics of the system, that is, how quickly the boats are traveling and how much the harmonics have changed within a given snapshot. The amplitude and background noise are then averaged over the length of the track to give the harmonic spectrum.

For the environment where the data presented in the paper was collected, the boats had to carefully maneuver their way out of Sequim Bay, so the velocity of the boat was not a real issue. However, the boats had a tendency to not stay at a constant velocity, thus the harmonics could spread across multiple frequency bins in any given snapshot. The size of the window to search for peaks was maximized for performance given that the peak could be a few frequency bins off of the projected frequency.

\section{DATA RESULTS}

The Sequim campus of PNNL is located at the mouth of Sequim Bay on the northern part of the Olympic Peninsula of Washington. The John Wayne Marina located inside the bay allows for a very diverse population of boat traffic coming in and out of the area. Thus it is an excellent location for collecting test data from different types of small to mid scale boats. PNNL has been continuously monitoring boat traffic 
at this site for almost two years, and provided a data set including passes of 50 boats for testing the HEAT algorithm. Many of the 50 boats are duplicate passes by the same or a similar type of boat. This allows the evaluation of HEAT algorithm on repeated harmonic signatures.

The data set included both acoustic and non-acoustic information. The acoustic data was collected using a single hydrophone mounted approximately $20 \mathrm{ft}$ from the dock on the ocean floor (approximately $30 \mathrm{ft}$ deep). The hydrophone used to record the boat noise was cabled back to the PNNL dock. At the dock the data was pre-processed using an antialiasing filter with cut-off frequency of $2.5 \mathrm{kHz}$. The data was then sampled at $8 \mathrm{kHz}$ with 16-bit resolution.

Among the non-acoustic monitoring methods, there was a radar system providing an estimate of boat velocity, an electro-optic and infrared (EO/IR) camera recording a video of each boat pass, and a number of environmental sensors giving measurements of water temperature, current, etc. PNNL also provided records of each boat such as the hull material, engine type (e.g., inboard or outboard), and approximated length.

In Ref. 22, the HEAT algorithm has been applied to extract features from data recorded in Hawaii as well as simulated boat data. In 2010, the authors in collaboration with PNNL conducted another data collection in Sequim Bay. In this data set, a boat with known parameters and known RPM was recorded The data was processed using the HEAT algorithm as well as a manual analysis which is equivalent to calculating the fundamentals from the known boat parameters and the RPM and comparing it to the recorded data. These results were reported in Ref. 23 .

\section{A. Application of HEAT to data}

The HEAT algorithm was used to process all 50 boat recordings from PNNL as described in the previous section. In this section we present the feature extraction results of four boat passes which best represent the data set. Table II gives a summary of these boats. This table shows the boat identification by letter (A-D), the hull material and engine type (if known), the harmonic content parameter $\Psi$ from the HEAT algorithm, and the mean frequency of the track of best fit. The four boats chosen were all $6 \mathrm{~m}$ inboard boats. The objective for selecting these four boats was to discriminate the harmonic signatures between inboard engine boats of approximately the same length with different hull materials.

Table III gives the parameters used for the HEAT algorithm. The same parameters were used to process all the data from the three different locations. The data has a low pass filter with cutoff at $2.5 \mathrm{kHz}$. For this reason the analysis was

TABLE II. List of boats used to test the HEAT algorithm.

\begin{tabular}{lccccc}
\hline \hline Boat ID & $\begin{array}{c}\text { Approx. } \\
\text { Length }\end{array}$ & $\begin{array}{c}\text { Engine } \\
\text { Type }\end{array}$ & $\begin{array}{c}\text { Hull } \\
\text { Material }\end{array}$ & $\Psi$ & $\begin{array}{c}\text { Mean } \\
\gamma_{K F, k}\end{array}$ \\
\hline A & $6 \mathrm{~m}$ & Inboard & Fiberglass & 0.468 & 31.6 \\
B & $6 \mathrm{~m}$ & Inboard & Fiberglass & 0.400 & 32.4 \\
$\mathrm{C}$ & $6 \mathrm{~m}$ & Inboard & Unknown & 0.195 & 20.6 \\
$\mathrm{D}$ & $6 \mathrm{~m}$ & Inboard & Aluminum & 0.138 & 29.8 \\
\hline \hline
\end{tabular}

TABLE III. Parameters used in the HEAT algorithm.

\begin{tabular}{lc}
\hline \multicolumn{1}{c}{ Variable } & Value \\
\hline Snapshot Window, T & $1 \mathrm{~s}$ \\
Snapshot Overlap & $50 \%$ \\
Frequency Limits & 0 to $2000 \mathrm{~Hz}$ \\
Normalizing Window, W & $25 \mathrm{bins}$ \\
$\gamma$ Limits & 4.5 to $65 \mathrm{~Hz}$ \\
$\Delta \gamma$ & $0.025 \mathrm{~Hz}$ \\
$\lambda_{\gamma}$ & 0.09 \\
M of N & 2 of $3 \mathrm{snapshots}$ \\
$\chi^{2}$ & 3 \\
NF & $3 \mathrm{snapshots}$ \\
$W_{\gamma}$ & $0.5 \mathrm{~Hz}$ \\
Process Noise Covariances & \\
$Q_{\gamma}$ & $(2 \Delta \gamma)^{2}$ \\
$Q_{\gamma}$ & $(2 \Delta \gamma)^{2} / 10$ \\
$Q_{\rho}$ & 0.02 \\
Measurement Noise Covariances & \\
$R_{\gamma}$ & $(5 \Delta \gamma)^{2}$ \\
$R_{\rho}$ & 0.03 \\
\hline \hline
\end{tabular}

only performed for frequencies up to $2 \mathrm{kHz}$. This greatly reduces the dimension of the data to be analyzed which allows for faster processing.

Figure 4 shows the spectrograms of each boat and their harmonic amplitude signatures extracted by HEAT. Boats A and B (Figs. 4(a) and 4(c)) show the case where the harmonics are clearly visible in the spectrogram throughout the entire frequency band. Boats C and D (Figs. 4(e) and 4(g)) show the opposite case where there are limited visible harmonics on the spectrogram within the frequency band.

The harmonic amplitude signatures for boats A through D are shown in Figs. 4(b), 4(d), 4(f), and 4(h). The stem plots represent the amplitude of each harmonic and the local noise around each peak is represented as a solid line. The amplitudes are in $\mathrm{dB}$ relative to the weakest harmonic. This is done since depending on the distance of the boat from the hydrophone, or the speed of the boat, the absolute amplitudes can widely vary, but the relative amplitudes of all the harmonics should stay the same regardless. An alternative signature could be shown by dividing the amplitude by the noise and then convert to $\mathrm{dB}$ to give the signal to noise ratio (SNR), but by putting the noise curve on the amplitude signature plot, both are visible.

Comparing the spectrograms of all the boats to the harmonic amplitude signatures, and specifically observing the intensity of the harmonic lines relative to the background noise, there is relatively good qualitative agreement between what can be seen in the spectrogram and what the HEAT algorithm extracted. Recall that the $\Psi$ parameter was defined in (15) as a measure of how much harmonic content is visible in the spectrogram. In Table II boats A and B have higher $\Psi$ values than boats $C$ and $D$, which follows along with definition of the $\Psi$ parameter.

\section{SUMMARY AND FUTURE WORK}

Classification between the acoustic signature from different types of small boats is a relatively unexplored research 

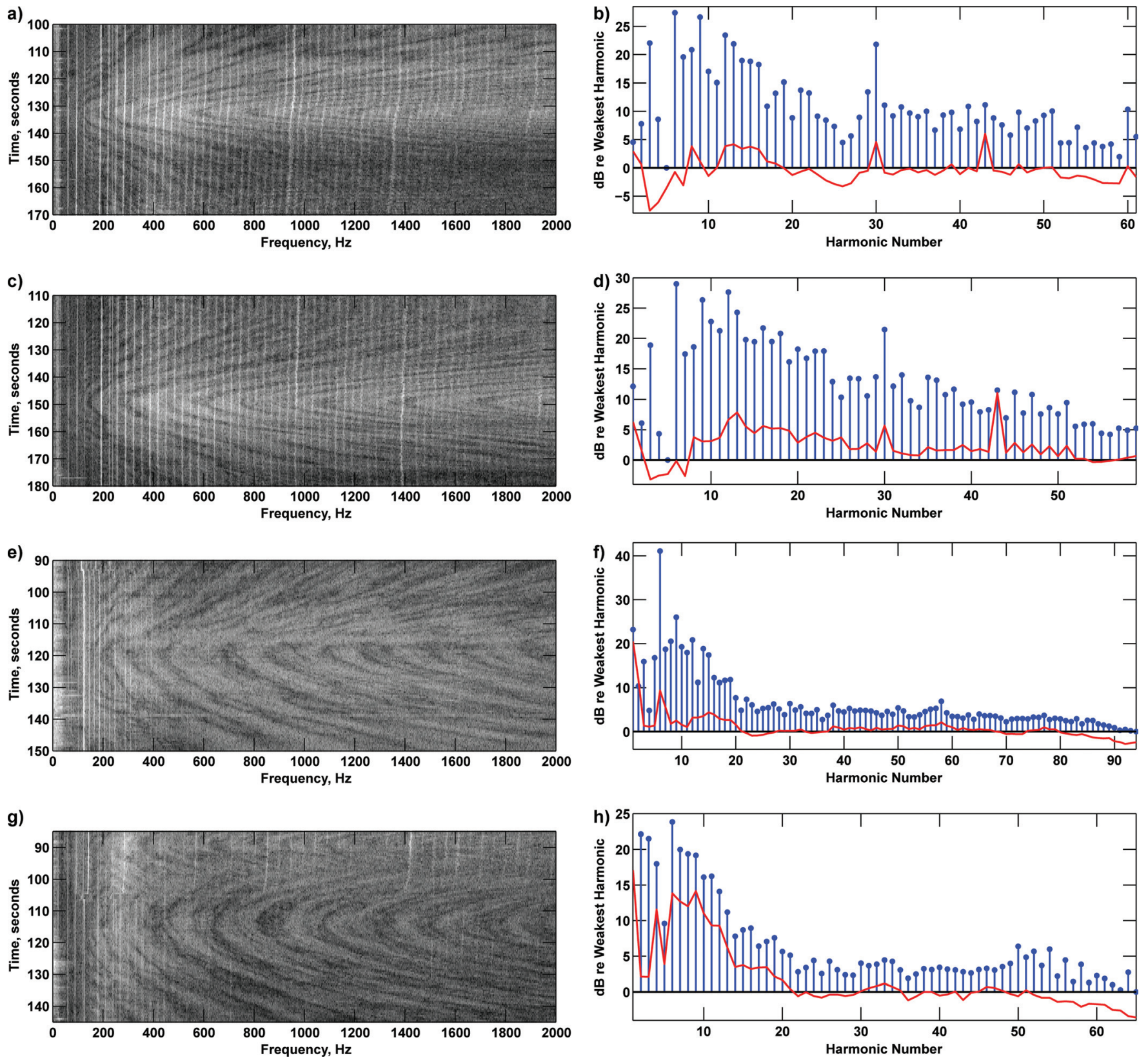

FIG. 4. (Color online) The figures on the left show the STFT of the data recorded on the hydrophone as boats A-D passed by. The figures on the right are the harmonic amplitude signatures (stem plot) with background noise (solid line) extracted from the STFT of each boat.

area. There has been a large amount of work on classification between large ships in the past, but for small boats there are limited efforts found in the literature. The goal of this paper is to develop a signal processing method to extract harmonic signatures in the noise radiated from small boats. Future work in this area includes testing the efficiency of these extracted signatures in the development of a harmonic-based classification scheme.

The signal processing algorithm developed in this paper extracts the amplitude of the harmonic content from the underwater noise generated by a boat using a combined likelihood and tracking approach. The likelihood provides an estimate of the fundamental frequency, and a Kalman filter is used to capture the time varying structure. These tracks of the fundamental frequency are then used to extract the amplitudes of the harmonics from the received data. The relative amplitudes of all the harmonics constitute a harmonic signature that can later be used in identification of the boats. The ability of the HEAT algorithm to estimate the fundamental and extract the harmonic information is demonstrated with a shallow water data set comprised of a number of boats with repeated occurrences. Preliminary results showing the robustness of the amplitude signature as quantified by a correlation analysis indicate the harmonics contain robust information related to the nature of the boat. Future research is needed to examine the harmonic output produced by the HEAT algorithm for different propagation environments and sensor geometries. Future research must also be done to evaluate the algorithms ability to separate harmonic content produced by multiple crafts passing the sensor. 
${ }^{1}$ M. McDonald and S. Lycett, "Fast versus slow scan radar operation for coherent small target detection in sea clutter," IEEE Proc. Radar Sonar Navig. 152, 429-435 (2005).

${ }^{2}$ S. P. Van Den Broek, H. Bouma, and M. A. Degache, "Discriminating small extended targets at sea from clutter and other classes of boats in infrared and visual light imagery," (SPIE, Orlando, FL, 2008) 69690B, Vol. 6969.

${ }^{3}$ D. Ross, Mechanics of Underwater Noise, (Pergamon Press, Los Altos, 1976), pp. 272-280.

${ }^{4}$ R. J. Urick, Principles of Underwater Sound, 3rd ed. (McGraw-Hill, New York, 1983), pp. 266-284.

${ }^{5}$ R. Stolkin, A. Sutin, S. Radhakrishnan, M. Bruno, B. Fullerton, A. Ekimov, and M. Raftery, "Feature based passive acoustic detection of underwater threats," volume 6204 (SPIE, Kissimmee, FL, United states), 620408, (2006).

${ }^{6}$ B. Borowski, A. Sutin, H.-S. Roh, and B. Bunin, "Passive acoustic threat detection in estuarine environments," (SPIE, Orlando, FL, 2008) 694513, Vol. 6945.

${ }^{7}$ G. Ogden, L. Zurk, M. Siderius, E. Sorensen, J. Meyers, S. Matzner, and M. Jones, "Frequency domain tracking of passive vessel harmonics," J. Acoust. Soc. Am. 126, 2249 (2009).

${ }^{8}$ Y. Medan, E. Yair, and D. Chazan, "Super resolution pitch determination of speech signals," IEEE Trans. Signal Process. 39, 40-48, (1991).

${ }^{9}$ A. Nehorai and B. Porat, "Adaptive comb filtering for harmonic signal enhancement," IEEE Trans. Acoust. Speech Signal Process. 34, 1124 1138 (1986).

${ }^{10} \mathrm{~S}$. Kim, L. Holmstrom, and J. McNames, "Multiharmonic tracking using marginalized particle filters," in Proceedings of the 30th Annual International Conference of the IEEE (August 20-25, 2008), pp. 29-33.

${ }^{11} \mathrm{C}$. Dubois and M. Davy, "Joint detection and tracking of time varying harmonic components: A flexible Bayesian approach," IEEE Trans. Audio Speech Language Process. 15, 1283-1295 (2007).
${ }^{12}$ H. Li, P. Stoica, and J. Li, "Computationally efficient parameter estimation for harmonic sinusoidal signals," Signal Process. 80, 1937-1944 (2000).

${ }^{13}$ M. G. Christensen, P. Stoica, A. Jakobsson, and S. H. Jensen, "Multi-pitch estimation," Signal Process. 88, 972-983 (2008).

${ }^{14}$ K. W. Chan and H. C. So, "Accurate frequency estimation for real harmonic sinusoids," IEEE Signal Process. Lett. 11, 609-612 (2004).

${ }^{15}$ M. S. Grewal and A. P. Andrews, Kalman Filtering: Theory and Practice (Prentice-Hall, Englewood Cliffs, NJ, 1993), Chap. 4, Sec. 4.2.

${ }^{16}$ S. P. Coraluppi and D. Grimmett, "Multistatic sonar tracking," Proc. SPIE 5096, 399-410 (2003).

${ }^{17}$ P. Meyer, R. Wilhelms, and H. W. Strube, "A quasiarticulatory speech synthesizer for German language running in real time," J. Acoust. Soc. Am. 86, 523-540 (1989).

${ }^{18}$ L. M. Gray and D. S. Greeley, "Source level model for propeller blade rate radiation for the world's merchant fleet," J. Acoust. Soc. Am. 67, 516-522 (1980).

${ }^{19} \mathrm{P}$. T. Arveson and D. J. Vendittis, "Radiated noise characteristics of a modern cargo ship," J. Acoust. Soc. Am. 107, 118-129 (2000).

${ }^{20}$ J. D. Wise, J. R. Caprio, and T. W. Parks, "Maximum likelihood pitch estimation," IEEE Trans. Acoustics Speech Signal Process. 24, 418-423 (1976).

${ }^{21}$ T. W. Anderson, An Introduction to Multivariate Statistical Analysis, 2nd ed. (Wiley-Interscience, New York, 1984,) p. 40-57.

${ }^{22}$ E. Sorensen, H. Ou, L. Zurk, and M. Siderius, "Passive acoustic sensing for detection of small vessels," Oceans (September 20-23, 2010) pp. 1-8.

${ }^{23}$ S. Matzner, A. Maxwell, J. Myers, K. Caviggia, J. Elster, M. Foley, M. Jones, G. Ogden, E. Sorensen, L. Zurk, J. Tagestad, A. Stephan, M. Peterson, and D. Bradley, "Small vessel contribution to underwater noise," Oceans (September 20-23, 2010) pp. 1-7. 